\title{
Examination of apparent extent as an explanation of the Poggendorff effect
}

\author{
M. T. TRUEMAN and A. E. WILSON \\ University of New Brunswick, Saint John, New Brunswick, Canada
}

\begin{abstract}
The explanation of apparent misalignment in the Poggendorff figure, based on underestimation of the intertransversal distance, was investigated in two experiments. In Experiment 1, subjects judged the intertransversal distance in the traditional Poggendorff figure and two of its variants. The size of the acute angle and the intertransversal distance were manipulated. Half of the subjects made the judgments with the method used by Wilson and Pressey (1976) and the other half made their judgments with the method used by Greist-Bousquet and Schiffman (1981). The results indicated that perceived intertransversal distance was greater with the former method. In Experiment 2, subjects adjusted the transversals to apparent collinearity in the same displays as were used in Experiment 1 . The collinearity judgments were transformed to allow comparison with the results of Experiment 1. Comparison of the collinearity judgments with the distance judgments indicated that they did not follow similar trends. For each Poggendorff variant, proportional distance judgments increased as the size of the acute angle increased, and decreased as the intertransversal distance increased. Collinearity judgments did not vary as a function of intertransversal distance. As the size of the acute angle increased, collinearity judgments increased for two of the Poggendorff variants but decreased for the third. It was concluded that the findings did not support the explanation of apparent misalignment based on underestimation of the intertransversal distance.
\end{abstract}

The traditional Poggendorff figure, as seen in Figure 1A, consists of a transversal separated by two vertical parallel lines. Although the two portions of the transversal are objectively collinear, subjectively they do not appear so. In the example shown in Figure 1A, the upper right transversal is judged higher than the linear extension of the lower left transversal. This apparent misalignment of the transversals is referred to as the Poggendorff effect.

Judd (1899) was one of the first investigators to discard the popular belief that the Poggendorff effect was one of direction and instead proposed that it was caused by a misjudgment of linear extent or distance. Specifically, Judd suggested that the perceived noncollinearity of the transversals was due to underestimation of the intertransversal distance. The underestimation, according to Judd, was caused by the influence of the components of the Müller-Lyer figure found within the Poggendorff display. As seen in Figures $1 \mathrm{~B}$ and $1 \mathrm{C}$, versions of the wings-out and wings-in Müller-Lyer figures are found in truncated variations of the traditional Poggendorff display.

By studying these variations of the Poggendorff figure, Judd (1899) discovered that the wings-in variation resulted in a stronger effect than the wings-out variation. Because

This research is based on a Master's thesis submitted to the University of New Brunswick by M. T. Trueman. The research was sponsored by the Natural Sciences and Engineering Research Council of Canada (A8323). Requests for reprints should be directed to A. E. Wilson, Division of Social Sciences, University of New Brunswick, Saint John, New Brunswick E2L 4L5, Canada. the wings-in variation had the greater influence, and because the wings-in Müller-Lyer figure induces underestimation, Judd concluded that the Poggendorff effect was caused by underestimation of the intertransversal distance. Subsequent research has confirmed that the wings-in variant induces a strong Poggendorff effect and that the wings-out variant produces either a small (Day, 1973) or a reversed effect (Day, 1973; Greist-Bousquet \& Schiffman, 1985; Houck \& Mefford, 1973; Restle, 1969).

Judd's (1899) distance theory has been assessed by means of requiring subjects to make judgments of the distance between the points of intersection of the transversals with the parallel lines--the intertransversal distance. The general prediction is that in variants of the Poggendorff figure that induce the classical misalignment effect, the distance will be underestimated, and that in variants that induce a reversed effect, the distance will be overestimated. As predicted, the wings-out variant (Figure 1B) induces overestimation of the intertransversal distance, and the wings-in variant (Figure 1C) generates underestimation of it (Greist-Bousquet \& Schiffman, 1981; Quina-Holland, 1977). However, findings made with the traditional Poggendorff display (Figure 1A) have been inconsistent. Pierce (1901) and Wilson and Pressey (1976) found overestimation, whereas Quina-Holland (1977) and Greist-Bousquet and Schiffman (1981) found underestimation. Another study found both overestimation and underestimation (Finlay \& Caelli, 1975).

Recently, Greist-Bousquet and Schiffman (1985) attempted to provide further support for Judd's distance theory. They compared the effects of varying the compo- 
A

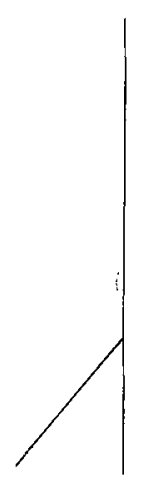

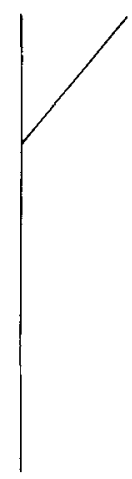

B

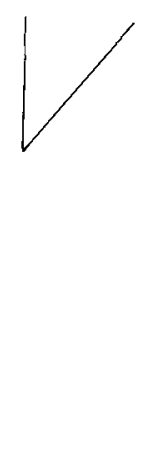



C

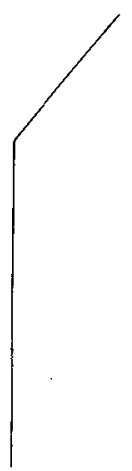

Figure 1. Examples of the traditional (A), wings-out, (B), and wings-in (C) variants of the Poggendorff figure.

nents within Poggendorff variants on judgments of collinearity with previous research conducted by Restle and Decker (1977) on the effects of varying analogous components within Müller-Lyer figures on judgment of apparent length. On the basis of Judd's theory, it was predicted that manipulation of analogous components would result in similar trends. Greist-Bousquet and Schiffman noted that the trends found with the traditional Poggendorff figure and the wings-in variant were generally consistent with the results of Restle and Decker. With the wings-out Poggendorff variant, however, the trends were not the same as those found by Restle and Decker. With this Poggendorff variant, the magnitude of the reversed illusion of alignment diminished as the size of the angle increased; but with the Müller-Lyer display, the apparent shaft length increased as the angle increased.

Although such comparisons between collinearity judgments with Poggendorff variants and judgments of linear extent with Müller-Lyer displays provide some indication of how the two types of judgments covary, a more exact assessment would be made if subjects were required to make both types of judgments with identical displays. Complementary results from judgments of distance and collinearity would provide strong support for Judd's distance theory. Therefore, the major aim of the present study was to provide further evaluation of distance theory by comparing judgments of distance and collinearity made with the same displays.

In Experiment 1, subjects were required to judge the intertransversal extent. The variables manipulated were the angle formed between the parallel components and the intertransversal extent, the intertransversal distance, and the variant of the Poggendorff figure. The Poggendorff variants included the traditional figure, the wings-in figure, and the wings-out figure. In Experiment 2, the subjects made judgments of collinearity. The displays and the variables manipulated were the same as in Experiment 1.

In Experiment 1, findings from two different methods of obtaining distance judgments were compared-the methods of Wilson and Pressey (1976) and GreistBousquet and Schiffman (1981), which previously have yielded opposing results. Using their respective techniques, Wilson and Pressey (1976) found overestimation and Greist-Bousquet and Schiffman (1981) found underestimation of the intertransversal distance. They had used displays of different dimensions, so the differences in their findings may have resulted from either the displays or the methods. By using both techniques with the same figures, we could assess the influence of method.

The method of Wilson and Pressey (which will henceforth be called the Wilson and Pressey method) requires the subject to first view the stimulus figure, which is next removed, and then reproduce the estimated intertransversal distance by placing a dot at a point horizontal to a single response dot. The method used by Greist-Bousquet and Schiffman (which will be referred to as the GreistBousquet and Schiffman method) also requires the subject to place a dot at an estimated distance from the response dot equal to the intertransversal extent. The methods differ, however, because with the GreistBousquet and Schiffman method, the response dot appears on the same sheet as the stimulus figure and the subject is required to maintain the same spatial orientation between the dots as in the stimulus figure.

\section{EXPERIMENT 1}

\section{Method}

Subjects. Eighty subjects from introductory psychology courses at the University of New Brunswick at Saint John participated in the experiment for course credit. Only those with normal or corrected-to-normal vision were asked to participate.

Design. A $3 \times 3 \times 3 \times 2$ mixed design was used. The withinsubjects variables were Poggendorff variation (wings-in, wings-out, and traditional figure), the angle between the intertransversal extent and parallel components $\left(22.5^{\circ}, 45^{\circ}\right.$, and $67.5^{\circ}$ for the wings-in and full figure and $157.5^{\circ}, 135^{\circ}$, and $112.5^{\circ}$ for the wings-out figure), and intertransversal distance $(17.5,35$, and $70 \mathrm{~mm})$. The between-subjects variable, which is described below in the procedure, comprised the two methods used to obtain distance judgments. For ease of description, the angle measurements for all Poggendorff variations will henceforth be discussed in terms of the acute angle within the figures. The acute angle for the wings-in and full figures remains the angle formed between the parallel components 
and the intertransversal extent, whereas the acute angle of the wingsout figure is the complement of the exterior angle formed between the parallel components and the transversal. An equal number of subjects (40) was randomly assigned to perform judgments using either the Greist-Bousquet and Schiffman or the Wilson and Pressey method.

Stimuli. Thirty-six figures ( 27 experimental and 9 control) were drawn with black ink on $21.6 \times 27.9 \mathrm{~cm}$ white sheets of paper and then reproduced for testing. Each experimental figure consisted of 35-mm vertical parallel components and two 35-mm collinear transversals. The control figures consisted of the collinear transversals. The width of each line was $.5 \mathrm{~mm}$. The vertical lines of the displays were oriented parallel to the narrow sides of the sheets of paper. The parallel line on the right side of the figure was positioned $100 \mathrm{~mm}$ from the left edge of the paper. A dot $1.5 \mathrm{~mm}$ in diameter, positioned $60 \mathrm{~mm}$ to the right of the right parallel line, served as the starting dot for the Greist-Bousquet and Schiffman method.

The response sheets for the Wilson and Pressey method consisted of a single $1.5-\mathrm{mm}$ dot on an otherwise blank sheet of paper. The position of the dot was adjusted for its corresponding stimulus figure, so that with a veridical judgment of intertransversal extent the horizontal distance between the provided dot and the response dot made by a subject would be an equal distance from each of the two parallel lines in the experimental figure.

Apparatus. Each stimulus figure was placed on a $45.2 \times 46.2 \mathrm{~cm}$ white target holder, which was tilted backwards $20^{\circ}$ so that the subjects viewed the displays perpendicularly. A chinrest was located directly in front of the holder, so that the viewing distance from the subject's eyes to the stimulus figure was uniform at approximately $50 \mathrm{~cm}$.

Procedure. The subjects were first informed of their rights as research participants. After completing a consent form, they were presented with sample display sheets and given instructions on how to do the task according to the method assigned to them.

The subjects making distance judgments with the Wilson and Pressey method were shown, on the sample display sheets, which distances they were to judge. They were then told that they would have $5 \mathrm{sec}$ to view each test display and judge the distance before the display was removed and replaced with the response sheet. It was explained that once the response sheet was in place, they should place a dot horizontally to the right of the response dot, at a point that appeared equal to the judged distance. The subjects were permitted to change their original choices if they wished; they were asked to indicate their final choices by circling them. Such changes, however, were infrequent.

The subjects using the Greist-Bousquet and Schiffman method were also shown, on display sheets, which distances they were to judge. They were then told that they would have $5 \mathrm{sec}$ to view each display and judge the distance before they would be informed to reproduce the judged distance relative to the response dot, which appeared to the right of the figure. It was explained and demonstrated on the display sheets that the distance and the spatial orientation of the judged distance should be reproduced when the dots were positioned. The subjects were permitted to change their original responses if they wished, and they were asked to indicate their final choices by circling them. Again, such changes were infrequent.

After the instructions were explained, the subjects were presented with two random sequences of the 36 experimental and control displays, which resulted in each subject's making 72 judgments. Each subject was given different randomized sequences of the displays.

\section{Results}

The distance between the center of each response dot and the center of each dot drawn by the subject was measured with a straightedge scale to the nearest $.5 \mathrm{~mm}$.
Proportional distance judgments (Greist-Bousquet \& Schiffman, 1985) were then calculated by means of the subtraction of the intertransversal distance judgment of the control figure from the intertransversal distance judgment of the experimental figure, and then the division of this difference by the actual intertransversal distance. Positive scores reflected overestimation of the objective distance, and negative scores indicated underestimation of the objective distance in the experimental figures.

The two proportional judgments for each stimulus figure were averaged and then submitted to a $2 \times 3 \times 3 \times 3$ (method $\times$ angle $\times$ distance $\times$ Poggendorff variation) mixed analysis of variance (ANOVA). The ANOVA indicated a significant main effect of method $[F(1,78)=$ $4.29, p<.05]$. On average, the subjects made longer proportional responses with the Wilson and Pressey method $(M=0.026)$ than with the Greist-Bousquet and Schiffman method $(M=.001)$. To determine if judgments for the traditional Poggendorff variation significantly overestimated or underestimated the distance with the two methods, $t$ tests, with alpha set at the .01 level, were conducted. A summary of the tests is presented in Table 1 . The summary indicates that the pattern of statistically significant results was similar for both methods. Significant underestimation was generally found when the intertransversal distance was longest $(70 \mathrm{~mm})$, regardless of the size of angle, and significant overestimation was found when the size of the acute angle was largest $\left(67.5^{\circ}\right)$ and the intertransversal distance was at either the smallest or the intermediate level.

The ANOVA also indicated that a significant three-way interaction among angle, distance, and Poggendorff variation occurred $[F(8,624)=4.79, p<.001]$. Figure 2, which combines the data obtained with both methods, depicts three basic trends in the data: (1) as the intertransversal distance increased, proportional judgments decreased; (2) as the size of the acute angle increased, proportional judgments increased; and (3) proportional judgments for the wings-out figures were longer than judgments for either the wings-in or full figure. Multiple comparisons made with Dunn's test (Keppel, 1973) verified

Table 1

$T$ Values of $t$ Tests Performed on Proportional Distance Judgments of the Traditional Poggendorff Variation Using Different Methods

\begin{tabular}{cccc}
\hline \multirow{2}{*}{$\begin{array}{c}\text { Intertransversal } \\
\text { Distance (mm) }\end{array}$} & \multicolumn{3}{c}{ Acute Angle } \\
\cline { 2 - 4 } & $22.5^{\circ}$ & $45^{\circ}$ & $67.5^{\circ}$ \\
\hline \multicolumn{4}{c}{ Wilson and Pressey Method } \\
17.5 & -0.19 & 1.81 & $3.54^{*}$ \\
75 & 0.91 & -0.27 & $3.96^{*}$ \\
70 & $-3.91^{*}$ & $-5.80^{*}$ & -1.04 \\
\multicolumn{4}{c}{ Greist-Bousquet and Schiffman Method } \\
17.5 & 0.35 & 1.45 & $4.73^{*}$ \\
35 & -0.44 & 0.46 & $3.08^{*}$ \\
70 & $-7.12^{*}$ & $-4.73^{*}$ & $-2.94^{*}$ \\
\hline
\end{tabular}

Note-Negative values indicate underestimations and positive values indicate overestimations. $\quad{ }^{*} p<.01 ; d f=39$. 

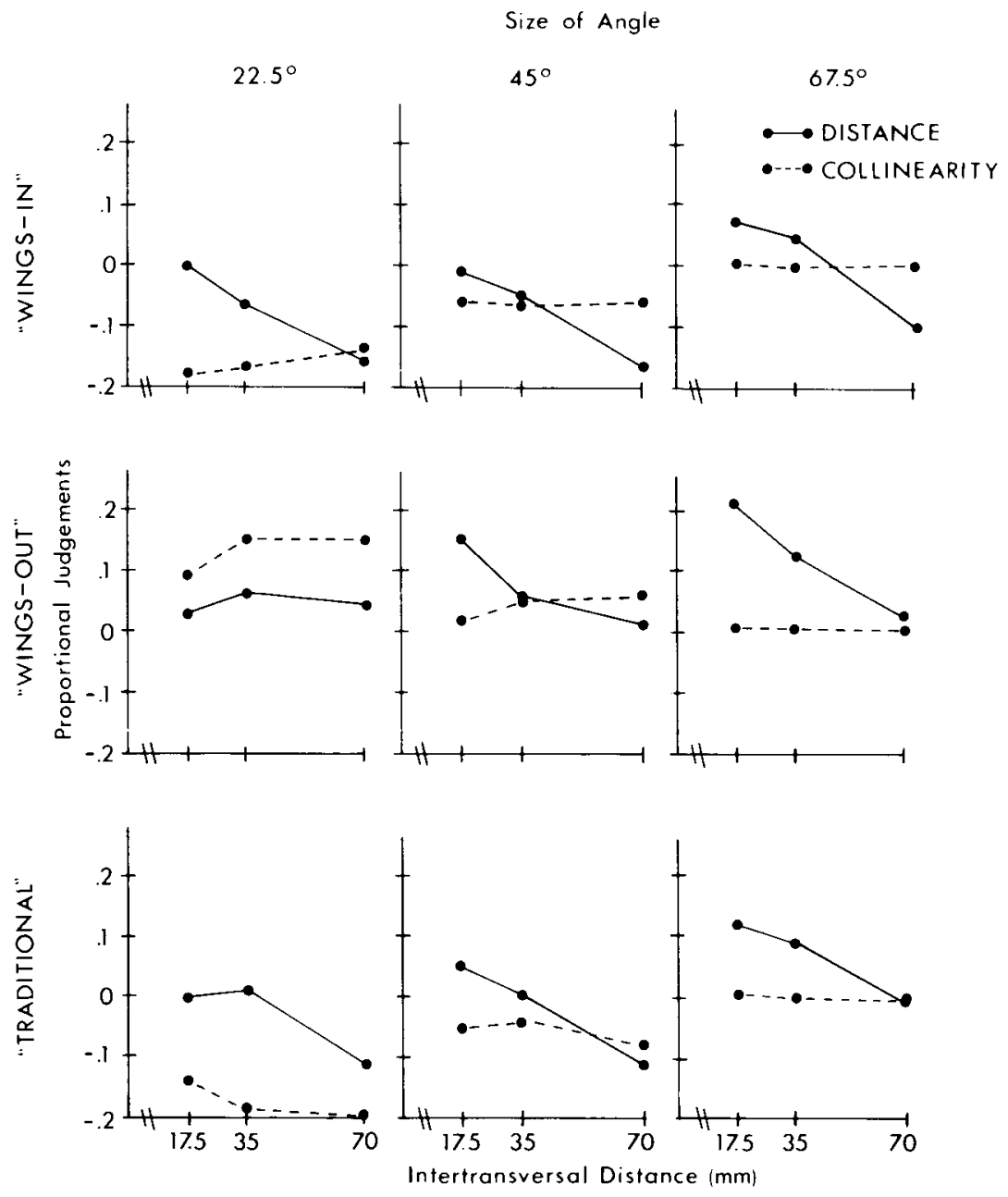

Figure 2. Proportional distance judgments (Experiment 1) and proportional collinearity judgments (Experiment 2) in the three Poggendorff figures as a function of size of acute angle and intertransversal distance.

that the proportional judgments for the wings-out variation $(M=0.082)$ were significantly longer than those for the traditional variation $(M=0.002, p<.001)$, which in turn were significantly longer than those of the wingsin variation $(M=-0.045, p<.001)$. To determine whether the proportional judgments produced by each variation were either underestimations or overestimations of the intertransversal distance, $t$ tests with alpha set at .01 were performed. It was discovered that for the Wilson and Pressey method, the wings-out figure produced significant overestimation of the intertransversal distance $[t(39)=7.62, p<.01]$. Both the underestimation produced by the wings-in figure and the overestimation produced by the traditional figure did not reach significance. For the Greist-Bousquet and Schiffman method, the wings-in figure induced significant underestimation $[t(39)=-6.50, p<.01]$, while the wings-out figure produced significant overestimation of the intertransversal distance $[t(39)=6.40, p<.01]$. The traditional figure was found to produce nonsignificant overestimation.

To investigate the three-way interaction further, separate method $x$ length $x$ angle ANOVAs were conducted on the judgments made for each Poggendorff variation. The ANOVAs indicated that for each variation, the main effects of angle and intertransversal distance were statistically significant $(p<.001)$, and that the main effect of method was statistically significant for the wingsin and wings-out variations $(p<.05)$. Also, a statistically significant interaction between angle and distance was found for the wings-out figure $[F(4,312)=10.73$, $p<.001]$.

The two-way interaction found in the ANOVA of the wings-out figure indicated the source of the three-way interaction among angle, intertransversal distance, and figure. As shown in Figure 2, proportional judgments of wings-out figures with $22.5^{\circ}$ angles were not affected by varying the intertransversal distance, whereas judgments 
of figures with $45^{\circ}$ and $67.5^{\circ}$ angles decreased as the intertransversal distance increased.

\section{Discussion}

It was discovered in Experiment 1 that measurements of intertransversal distance using different methods but similar displays resulted in common findings, implying that the discrepant results found by Wilson and Pressey (1976) and Greist-Bousquet and Schiffman (1981) were largely due to the use of different displays. The analysis of the responses made with the traditional figure revealed that overestimation and underestimation of the intertransversal distance was dependent on the specific parameters of the display rather than on method. More specifically, overestimation occurred with the largest acute angle and underestimation resulted with the longest intertransversal distance. Overestimation with the traditional figure creates problems for distance theory, which clearly predicts underestimation of the intertransversal distance for the traditional Poggendorff figure.

The significant main effect of method indicated that the Wilson and Pressey method generated judgments of longer intertransversal distance than did the Greist-Bousquet and Schiffman method for each Poggendorff variation. However, the results of the $t$ tests comparing the means of the Poggendorff variations for each method, as well as the nonsignificant interactions among method and the other variables, suggest that the effect of the method pertained only to the degree of overestimation or underestimation rather than to the occurrence of either.

There were two basic differences between the two methods, which may explain why the Wilson and Pressey method produced longer proportional distance judgments. With the Wilson and Pressey method, subjects placed their responses horizontally, whereas with the Greist-Bousquet and Schiffman method, subjects placed their responses at an angle, in order to maintain the same spatial relationship as depicted in the stimulus figure. Positioning the response at an angle may have induced shorter proportional distance judgments than when producing the response horizontally. Another difference was that the stimulus figure was removed after $5 \mathrm{sec}$ of viewing with the Wilson and Pressey method, thus requiring the subjects to rely on memory while making their responses. It is possible that without a reference, subjects tended to overestimate the judged distance. However, it should be kept in mind that both of these possibilities have to be assessed in terms of differences relative to control judgments. It seems unlikely that either explanation could account for the effect of method.

Multiple comparisons among Poggendorff variations indicated that mean proportional judgments for the wingsout figure were significantly longer than those with either the wings-in or full figure. These results support the prediction based on distance theory that the wings-out figure would generate different judgments significantly different from those for the full and wings-in figure. However, the further prediction that the wings-out figure would produce overestimation and that the wings-in and full figures would produce underestimation was only partly fulfilled, since the full figure induced slight overestimation. As stated earlier, distance theory is not entirely supported by the results.

\section{EXPERIMENT 2}

In Experiment 2, collinearity judgments were obtained with the same displays as those used in Experiment 1. The subjects made their judgments by adjusting the right transversal so that it appeared collinear with the left transversal. On the basis of distance theory, it was predicted that proportional collinearity judgments would follow trends similar to those of the proportional distance judgments found in Experiment 1 .

\section{Method}

Subjects. Forty-eight subjects from introductory psychology courses at the University of New Brunswick at Saint John participated in the experiment for course credit. Only those with normal or corrected-to-normal vision were asked to participate.

Design. A $3 \times 3 \times 3$ mixed design was used. The within-subjects variables were the angle formed between the intertransversal extent and parallel components, and the intertransversal distance. The between-subjects variable was the variation of the Poggendorff figure. An equal number of subjects (16) was randomly assigned to make collinearity judgments, using either the wings-in, wingsout, or full figure.

Stimuli. The stimulus figures were photocopied on $27.9 \times 42.5 \mathrm{~cm}$ sheets of white paper. The sheets of paper were then cut vertically in half, midway between the transversals. All the display sheets contained a dot to the right of the figure, to ensure identical displays in both experiments.

Apparatus. The apparatus consisted of two adjacent segments of plywood, mounted inside a wooden box $(89 \times 89 \times 8 \mathrm{~cm})$. The left segment $(23 \times 29.3 \mathrm{~cm})$ was fixed, but the right segment $(23 \times 56 \mathrm{~cm})$ could be moved vertically if one turned an attached crank that extended beyond the frame of the box. A wooden rod was attached to the crank, allowing for easy manipulation from a sitting position. A wooden board with a circular viewing aperture (26.7 $\mathrm{cm}$ in diameter) covered the entire surface of the box. The board was attached by hinges, which made it possible for the experimenter to open and close the box in order to locate the stimulus sheets and measure the responses. The two halves of the stimulus figure were placed on the adjacent segments inside the box, so that they could be seen through the aperture when the box was covered. Inside the box, a ruler was attached adjacent to the movable right-hand segment, so that the experimenter could measure how far the right half of the stimulus sheet had been adjusted relative to the point of objective collinearity. The entire box was mounted $81.5 \mathrm{~cm}$ from the floor and tilted backwards $20^{\circ}$. A chinrest was positioned so that the viewing distance from the subject's eyes to the stimulus figure was uniform, approximately $50 \mathrm{~cm}$.

Procedure. The subjects were first informed of their rights as research participants and then asked to complete a consent form. They were shown sample displays and told that their task was to adjust the right transversal to apparent collinearity with the left transversal. Each subject was tested on 18 displays ( 9 experimental plus 9 control figures), which were presented in random order. The subjects made four consecutive adjustments for each figure, resulting in a total of 72 adjustments. Prior to each adjustment, the right transversal was placed randomly between 5 and $6 \mathrm{~cm}$ too high or too low from the objective point of collinearity by the experimenter. 
Placing the transversal too high or too low was also chosen randomly in pairs of two, so that each figure always appeared too high twice and too low twice regardless of order.

\section{Results and Discussion}

Initially, linear displacement was measured with a straightedge to the nearest $.5 \mathrm{~mm}$. Intertransversal distance was then determined by using trigonometry. The four measurements for each figure were averaged, and proportional judgments (Greist-Bousquet \& Schiffman, 1985) were then calculated by means of the subtraction of the average intertransversal distance judgment of the control figure from the average of the intertransversal distance judgments of the experimental figure, and then the division of this difference by the actual intertransversal distance. As in Experiment 1, positive scores reflected overestimation of the intertransversal distance and negative scores indicated underestimation.

The proportional judgments were then submitted to a $3 \times 3 \times 3$ (Poggendorff variation $\times$ angle $\times$ distance) mixed-effects ANOVA. The ANOVA indicated a significant main effect of Poggendorff variation $[F(2,45)=$ $66.01, p<.001]$. Multiple comparisons made with Dunn's test (Keppel, 1973) showed that proportional judgments for the wings-out figure $(M=0.059)$ were significantly longer $(p<.001)$ than those for both the wings-in $(M=-0.076)$ and the full figure $(M=-0.080)$. There was no significant difference between the judgments made with the wings-in and with the full figures. To determine whether the proportional judgments of each figure were significantly overestimated or underestimated, $t$ tests, with alpha set at .01 , were performed. It was found that the wings-out figure induced significant overestimation $[t(15)=4.92, p<.01]$, and that the wings-in and full figures induced significant underestimation $[t(15)=-8.44, p<.01$, and $t(15)=$ $-10.00, p<.01$, respectively].

The ANOVA also indicated a significant main effect of angle $[F(2,90)=23.18, p<.001]$ and a significant interaction between angle and Poggendorff variation $[F(4,90)=45.46, p<.001]$. From Figure 2 it is clear that as the size of the acute angle increased, proportional judgments for the wings-in and traditional figures increased, whereas those for the wings-out figure decreased. This figure also indicates that as intertransversal distance increased, proportional judgments varied only slightly.

\section{GENERAL DISCUSSION}

The major aim of this study was to assess Judd's (1899) distance explanation of the Poggendorff illusion by obtaining estimates of perceived intertransversal extent and apparent collinearity with identical displays. The general prediction was that both types of judgment would show similar trends. Figure 2 provides a direct assessment of the prediction. From the figure it is clear that proportional distance judgments were overestimates for the wings-out variant and underestimates for the wings-in display. For the traditional Poggendorff figure, both overestimation and underestimation occurred. Proportional collinearity judgments were overestimates for the wings-out variant and underestimates for the wings-in and traditional figures.

It is also clear from Figure 2 that the trends for proportional distance and collinearity judgments differed as a function of intertransversal distance and acute angle. Proportional distance judgments decreased as a function of intertransversal distance and increased as a function of acute angle. Proportional collinearity judgments, however, did not vary as a function of intertransversal distance, but were found to increase as a function of acute angle for the wings-in and traditional figures and to decrease as a function of acute angle for the wings-out figure. These findings indicate that a theory based on underestimation of the intertransversal distance does not provide an adequate explanation of the traditional Poggendorff effect. This conclusion is consistent with the outcome of a recent study by Day, Jolly, and Duffy (1987). With displays and procedures different from those used in the present study, they too concluded that apparent alignment and apparent extent in Poggendorff displays do not covary.

Distance explanations based upon extents other than the intertransversal could be explored. For example, Day and Dickinson (1976) argue that the vertical distance between transversals and the horizontal extent between the parallel lines are misjudged and lead to the apparent misalignment of the transversals. Further research into other explanations, such as that suggested by Day and Dickinson (1976), must be conducted before misjudgment of distances within the Poggendorff figure can be dismissed as a viable explanation of the Poggendorff illusion.

\section{REFERENCES}

DAY, R. H. (1973). The Poggendorff illusion with obtuse and acute angles. Perception and Psychophysics, 14, 590-596.

DAY, R. H. \& Dickinson, R. G. (1976). The components of the Poggendorff illusion. British Journal of Psychology, 67, 537-552.

DAY, R. H., Jolly, W. J., \& DuFF, F. M. (1987). No evidence for apparent extent between parallels as the basis of the Poggendorff effect. Perception \& Psychophysics, 42, 561-568.

Finlay, D. C., \& CAELL, T. M. (1975). The Poggendorff illusion and estimates of the transverse extent. Perceptual \& Motor Skills, 41, 143-148.

Greist-Bousquet, S., \& Schiffman, H. R. (1981). The Poggendorff illusion: An illusion of linear extent? Perception, 10, 155-164.

Greist-Bousquet, S., SChIFFMAN, H. R. (1985). Poggendorff and Müller-Lyer illusions: Common effects. Perception, 14, 427-447.

Houck, R. L., \& Mefroko, R. B. (1973). The Poggendorff illusion: A neurophysiologically based hypothesis. American Joumal of Psychology, 86, 283-309.

JuDD, C. H. (1899). A study of geometrical illusions. Psychological Review, 6, 241-261.

KEPPEL, G. (1973). Design and analysis: A researcher's handbook. Englewood Cliffs, NJ: Prentice-Hall. 
PierCe, A. H. (1901). Studies in auditory and visual space perception. New York: Longmans Green.

Quina-Holland, K. (1977). Spatial distortions within the Poggendorff figure and its variants: A parametric analysis. Perception \& Psychophysics, 21, 118-124.

Restle, F. (1969). Illusions of bent line. Perception \& Psychophysics, 5, 273-274.

Restle, F., Decker, J. (1977). Size of the Mueller-Lyer illusion as a function of its dimensions: Theory and data. Perception \& Psychophysics, 21, 489-503.

Wilson, A. E., \& Pressey, A. W. (1976). The role of apparent distance in the Poggendorff illusion. Perception \& Psychophysics, 20, 309-316.

(Manuscript received August 30, 1988;

revision accepted for publication November 10, 1988.)

\section{Announcement}

\section{4th Annual Boston University Conference on Language Development October 13, 14, and 15, 1989}

The 14th Annual Boston University Conference on Language Development is scheduled for October 13, 14 , and 15,1989 . The keynote speaker will be William Labov of the University of Pennsylvania.

Sessions will focus on the following central themes: American Sign Language; Literacy in Theory and in Practice (literacy and ideology, literacy at school, in the humanities, in the workplace, minorities' interpretation, sociocultural approaches); Language Acquisition (theories of evidence, neural maturation); First Language Acquisition (including principles and parameters, acquisition of LF, irregular phenomena, phonology); and Second Language Acquisition (including age factors, parameter setting, and sociocultural factors).

For further information and a preliminary program, please contact Conference Committee, Conference on Language Development, Boston University, 605 Commonwealth Ave., Boston, MA 02215 (telephone: 617-353-3085). 